\title{
The Interpretation of Trends in Treatment Statistics: An Example from Norway
}

\author{
A. John Spencer* \\ Brian A. Burt** \\ Per Baerum $\dagger$ \\ Ingolf Myrhaug†
}

Trends over time in the prevalence and severity of oral conditions are best established by periodic epidemiologic surveys of representative samples of the population, conducted at regular intervals by trained examiners using the same criteria and methods. However, few countries or public health jurisdictions have the resources even to attempt to reach this ideal, so simpler and cheaper compromises are frequently sought. The inelegant but inexpensive "pathfinder" surveys, encouraged by the World Health Organization, represent one such compromise which clearly has a place in obtaining epidemiological data for certain purposes. ${ }^{10}$

As another compromise, attention might be given to the use of treatment statistics as indicators of time trends in oral conditions. After all, many dental public health programs already collect treatment statistics, but all too often these statistics find little use beyond a mention in the agency's annual report. Perhaps the time has come to see if these data can be better used to discern epidemiological trends. Of course, it is traditionally accepted that dental treatment statistics cannot be used to discern epidemiological trends because of self-selected samples and the variability in the clinical dentists' approach to treatment plans. However, it may be that specific treatment statistics, when used under certain conditions, could be a valid and inexpensive source for discerning time trends in oral conditions.

As an example of the way that treatment statistics have been used to indicate time trends in oral conditions, Brudevold ${ }^{3}$ pointed to Norway as one of a few countries which has treatment data from large populations reflecting a successful preventive program. Such a casual claim, however, may or may not be valid. The purpose of this paper is to examine treatment statistics from Norway in order to highlight some possible difficulties in the interpretation of treatment statistics for discerning time trends in oral conditions. Some suggestions toward the development of more rigorous methods of collecting and analyzing treatment statistics will then be presented.

While Norwegian data are chosen as an example, it is not necessarily suggested that the way the data are collected and presented is typical of all dental public health programs, nor that difficulties in interpretation will be present in all situations.

\section{BACKGROUND}

Norway has long been considered a country with one of the greatest severities of

\footnotetext{
*Australian National Health and Medical Research Council Fellow in Applied Health Sciences, Department of Conservative Dentistry, Faculty of Dental Science, University of Melbourne, Melbourne, Victoria 3000, Australia.

**Associate Professor, Program in Dental Public Health, School of Public Health, The University of Michigan, Ann Arbor, Michigan 48109.

$\nmid$ Dental Division, Directorate of Health, Ministry of Social Affairs, Oslo-dep., Oslo 1, Norway.
}

[Submitted $2 / 28 / 80$; returned for revisions $3 / 14 / 80$; resubmitted $4 / 3 / 80$; accepted for publication $8 / 6 / 80$ ] 
dental caries in the world, ${ }^{5}$ and also has had a treatment service for school children for a considerable time through the School Dental Service (SDS)., ${ }^{1,2}$ The extent of treatment delivered by the SDS has been dependent on what the individual municipality decided and has had the financial means to make available. ${ }^{1}$ The SDS is currently being incorporated into the Public Dental Service (PDS) and its dental care delivery responsibilities are being taken over by the PDS. The PDS provides dental care free of charge to the 6-17 year-old age group, and to other age groups to some extent. Treatment is given in public clinics by district dental officers. District dental officers generally devote about 60 percent of their time to incremental care of children aged 6-17 years old. The PDS is fully developed in six counties, partially developed in another nine, and not yet introduced in four counties. ${ }^{1}$

Over the 1966 to 1978 period preventive programs developed markedly. Programs for rinsing or brushing with various fluoride vehicles have been established for schoolchildren, fluoride toothpastes have become widely used, and organized distribution of fluoride tablets for infants and older children has become widespread. ${ }^{1}$ Total sales of fluoride tablets, which no longer require a prescription, have greatly increased since 1971.1 A marked reduction in sugar sales in Norway has been observed though the permanency of this reduction is unknown. ${ }^{2}$ There has also been increased provision of preventive services in the clinical setting. ${ }^{1}$

\section{A FRAMEWORK FOR A CRITICAL APPRAISAL OF THE DATA AND THEIR INTERPRETATION}

The data examined in this paper are derived from the PDS and consist of:

(1) Selected treatment items provided per 100 persons aged 6-17 and treated through the PDS between 1966 and 1978 (Table I).

(2) Surfaces filled and dentists hours required per person 6-17 years old in the Snása district between 1972 and 1976 (Figure 1).

The trends observed from these data are a decrease in the number of selected treatment items associated with the sequelae of dental caries (both Table I and Figure 1), and an apparent lack of correlation in the decrease of the selected treatment items with the dental hours required per person (Figure 1).

In the public program from which these data are taken, a number of factors related to their internal and external validity, taken from the model presented by Campbell and Stanley, ${ }^{4}$ will be described and their possible confounding effects upon the interpretation of a causal effect in the data will be examined. ${ }^{4}$ The term internal validity, as used by Campbell and Stanley, is the extent to which the observation represents a true occurrence. External validity asks the question of generalizability of the trends observed. ${ }^{4}$

\section{INTERNAL VALIDITY}

History refers to events occurring during the period of data collection which could have influenced the trend observed. There were a number of such events in Norway during the 1966 to 1978 period. First, the organization of dental care system changed; most significantly the PDS began operating on an incremental care basis. ${ }^{1}$ Second, the PDS expanded considerably between 1966 and 1978; increases in the number of dentists employed, ${ }^{1}$ with consequent changes in the age, skill, and treatment philosophies of the dental work force, may have influenced the trends observed. Third, as use of dental services by persons 16 years old and over in Norway 
TABLE I

SELECTED DATA FROM ANNUAL SERVICE REPORTS OF THE PUBLIC DENTAL SERVICE IN INDIVIDUAL DISTRICTS AND COUNTIES, NORWAY

\begin{tabular}{clccc}
\hline & & \multicolumn{3}{c}{$\begin{array}{c}\text { Selected treatment items provided per } 100 \\
\text { persons-permanent dentition }\end{array}$} \\
\cline { 3 - 5 } & $\begin{array}{l}\text { Number of } \\
\text { persons seen } \\
6-17\end{array}$ & $\begin{array}{c}\text { Teeth extracted } \\
\text { Yecause of caries }\end{array}$ & $\begin{array}{c}\text { Rootfilled } \\
\text { teeth }\end{array}$ & $\begin{array}{c}\text { Tooth surfaces } \\
\text { filled }\end{array}$ \\
\hline 1966 & 127,700 & 8.0 & 1.2 & 617.9 \\
1967 & 140,594 & 7.6 & 1.0 & 599.9 \\
1968 & 136,598 & 7.1 & 1.1 & 626.0 \\
1969 & 149,037 & 6.4 & 1.1 & 607.9 \\
1970 & 164,082 & 5.3 & 1.1 & 608.9 \\
1971 & 189,363 & 4.4 & 1.0 & 608.0 \\
1972 & 217,134 & 3.2 & 0.9 & 546.4 \\
1973 & 244,507 & 2.2 & 0.8 & 473.2 \\
1974 & 274,698 & 1.6 & 0.7 & 414.7 \\
1975 & 296,839 & 1.0 & 0.6 & 373.7 \\
1976 & 322,875 & 0.7 & 0.5 & 340.5 \\
1977 & 343,000 & 0.6 & 0.4 & 308.8 \\
$1978 *$ & 366,525 & 0.4 & 0.5 & 280.6 \\
\hline
\end{tabular}

* Preliminary data.

Source: The Directorate of Health, Oslo, Norway.

FIGURE 1

TOOTH SURFACES FILLED AND DENTIST HOURS REQUIRED PER CHILD 6-17 YEARS OLD, PER YEAR. SNÄSA, NORWAY.

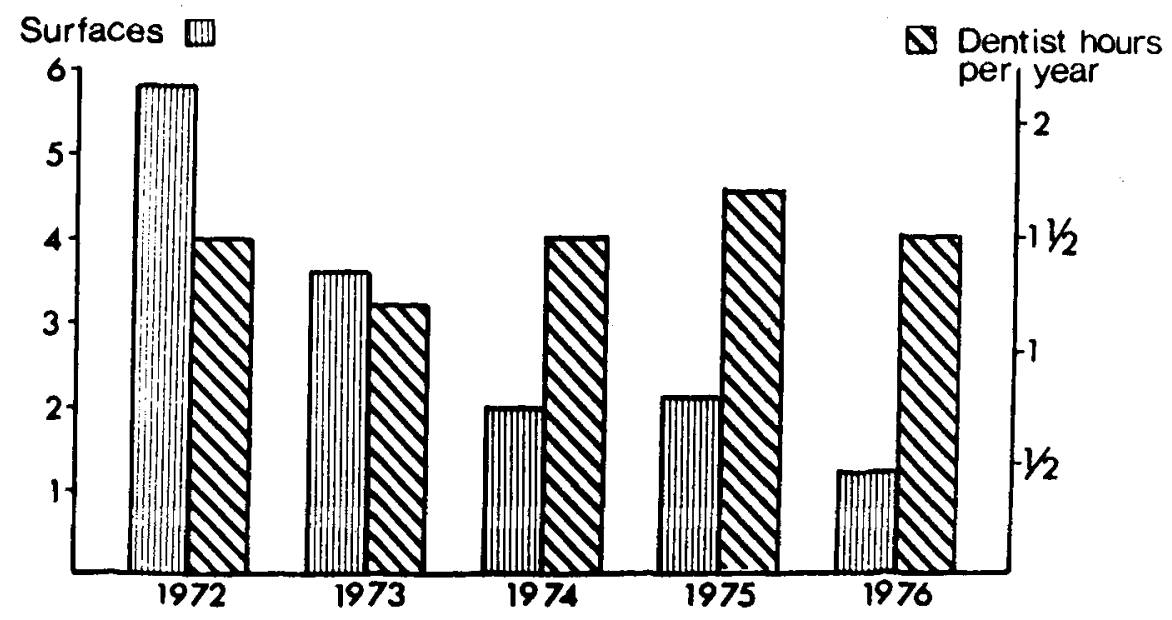

Source: Norwegian Directorate of Health 
has been increasing, 9 it is also likely (in the absence of definitive data) that the use of services by 6-17 year olds similarly increased over the period 1966 to 1978 .

Maturation refers to processes within the observed persons as a function of the passage of time per se. One obvious maturation possiblity is that the observed decrease in the selected treatment items per child (Table I) would naturally develop with the increasing proportion of children in the incremental program that required only maintenance care. ${ }^{7}$ However, while the increase in the number of $6-17$ year olds treated in the PDS (Table I) is consistent with the growth of an incremental plan, ${ }^{6}$ growth in coverage at all ages has occurred, ${ }^{1}$ presumably from the expansion of the PDS. It is not clear from the data how many of the persons seen in any one year are in the maintenance phase of an incremental care program, and how many are new to the program and may therefore have presented with a backlog of dental needs. If the proportion of 6-17 year olds seen each year that are new to the program has decreased over the 1966 to 1978 period, then it is likely that the proportion of those receiving maintenance care has increased; this factor may contribute to the trends shown in the treatment statistics.

Testing, the activity of collecting data per se, could affect the trends seen. While the collection of data within the PDS involves no activity separate from the normal delivery of care, the availability of the data may have led to alterations in the philosophy of care. A change in philosophy of care within the PDS to prevention rather than reparative treatment could have been spurred by the availability of treatment statistics. The increased delivery of preventive services is evidence of such an alteration in philosophy of care and may have been a factor in the lack of correlation between surfaces filled and dentist hours worked that may be observed in Figure 1. A change in philosophy of care is likely to be reflected in changes in diagnostic criteria and treatment planning.

Instrumentation refers to the possible influence of changes in examiners or diagnostic criteria over the period of data collection. Many different dentists were involved in providing treatment and in recording treatment statistics over the 1966 to 1978 period, and variable interpretation within and between dentists could easily have occurred. No specific criteria were used in the PDS to help standardize treatment planning or determination of hours worked in or out of the clinic.

Determination of the need for treatment is a subjective diagnostic judgment in the PDS as elsewhere. The outcome is sensitive to changes in the attitudes and behavior of both the providers and the recipients of dental care. The trend observed in Figure 1 could indicate shifts in attitude and behavior in both dentists and patients. Other treatment items, mostly likely diagnostic and preventive services, could be occupying more treatment time in the later years of the period.

There are several other factors, related to internal validity, which could conceivably have influenced the trends shown. For example, differential selection of patients in terms of age, sex, geographic location, parent education and income could contribute to the observed trends, because the importance of these factors in relation to dental health in Norway has been documented. ${ }^{1}$ Differential loss of patients through their failure to continue to use the service although eligible could also bias the data in favor of people with little need for the selected treatment items.

\section{EXTERNAL VALIDITY}

The data shown in Table I are taken from a high percentage, up to 90 percent, of 
all those children served by the PDS. ${ }^{3}$ The reason for exclusion of some children's records is not known. The proportion of the total child population's being treated by the PDS greatly increased during the 1966 to 1978 period. If there was differential selection of patients in the early years of the PDS, as mentioned previously, the data from the earlier years in Table I may have been biased, so that the observed trends could represent a regression phenomenon. The generalizability of the data to the whole Norwegian child population would therefore be reduced.

\section{DISCUSSION}

Campbell and Stanley's model is a useful one to follow in the critical appraisal of these Norwegian data. While one may intuitively agree with Brudevold ${ }^{3}$ that the preventive programs described may be producing beneficial results, it is clear from this appraisal that the observed trends could be due to other factors or a combination of factors. The development of the PDS as an incremental program, changes in dental treatment philosophy for whatever reason, the absence of standardized methods for collecting data, and possible differential selection and loss of patients may all influence the observed trends as much as the preventive program does. It is difficult to reach firmer conclusions from the data presented.

If treatment statistics are to be of use in discerning epidemiological trends, and in investigating intervention effects, they will need to be recorded in a more standardized pattern and the analysis of such data improved. From this appraisal of Norwegian data, several suggestions toward this end can be made. First, wherever possible, the treatment procedures being recorded should be well-defined and the recording methods clearly specified. Carefully developed and tested record forms of patient treatment seem a necessity. Such forms can help standardize necessarily subjective areas, such as treatment planning, by requiring all treating dentists to follow a common approach to diagnosis. This requirement does not mean pushing the clinical dentists into "cookbook" dental care, only that their approach to diagnosis and treatment planning be made systematic. New dentists and assistants in the service clearly need some training in the use of such record forms and the rationale behind them if this approach is to work. The procedure for recording dentists hours per child, and defining what time should be recorded under what category, is clearly an area that could be standardized by precise definition.

In the statistical analysis of the treatment data, control is required for as many factors as possible - for instance, analyzing data only from certain subregions in order to avoid those problems associated with the differential selection of patients over time. Further, those variables identified as possibly affecting the treatment statistics through concomitant variation could be statistically controlled during data analysis. For example, age and the number of previous treatment series received may be used to control for the problems associated with maturation of the incremental program. Indications of the directions that may be followed in design of experiments involving the collection of treatment statistics over time can be gained from Campbell and Stanley ${ }^{4}$ and Glass, Willson, and Gottman. ${ }^{8}$ Analysis of trends in treatment statistics may require use of multiple regression technics.

Retrospectively, no action can be taken to nullify the problems resulting from the fact that the Norwegian data shown were collected by many dentists over a 12-year period. Hence, more useful treatment statistics will be generated from programs like the PDS only if subsequent data collected are both standardized by the methods 
discussed, and possibly disaggregated to reveal different types of items, as well as supported by other types of sociodemographic data that can only be defined by an appraisal of all possible confounding factors. In this respect the data collection becomes more arduous, and it may be desirable to limit collection to representative groups of subregions and also to particular types of treatment that can be used as indicator variables regarding dental health. Representativeness of the patients from whom data are collected will also need to be considered.

In conclusion, more research is required to identify those treatment variables which are the best indicators of dental health, as well as to determine the most appropriate statistical methods for analysis. With progress in these areas, treatment statistics from public programs may fill a more useful role in discerning epidemiological trends and investigation intervention effects in dental programs.

\section{References}

1. Baerum, Per. Dental health and services in Norway. Oslo, Dental Division, Directorate of Health, 1978. 27 p.

2. . Dental care delivery in Norway. p. 125-35. (In Ingle, J.I., and Blair, Patricia, eds. International dental care delivery systems; issues in dental health policies. Cambridge, Mass., Ballinger, 1978. $\mathrm{xxi}+263 \mathrm{p}$.)

3. Brudevold, Finn. Broadening the mission of I.A.D.R. J. Dent. Res., 58, Special Issue C: 1180-3, Apr. 1979.

4. Campbell, D.T., and Stanley, J.C. Experimental and quasi-experimental designs for research. Chicago, Rand McNally, 1963. ix + 84 p. (p. 5,6,37- 42)

5. Cohen, Lois K. Dental care delivery in seven nations: the International Collaborative Study of Dental Manpower Systems in relation to Oral Health Status. p. 201-14. (In Ingle, J.I., and Blair, Patricia, eds. International dental care delivery systems; issues in dental health policies, Cambridge, Mass., Ballinger, 1978, xxi +263 p.)

6. Dunning, J.M. Principles of dental public health. 2nd ed. Cambridge, Mass., Harvard University Press, 1970. xiv +598 p. (p. 285)

7. Evans, R.G., and Williamson, M.F. Extending Canadian health insurance: options for pharmacare and denticare. Toronto, University Toronto Press, 1978. xii +276 p. (p. 113)

8. Glass, G.V., Willson, V.L., and Gottman, J.M. Design and analysis of time-series experiments. Boulder, Colorado, Colorado Associated University Press, 1975. xi +241 p.

9. Helöe, L.A. Changes of dental treatment patterns in Norway in the 1970s. Community Dent. Oral Epidemiol., 6:53-6, Mar. 1978.

10. Infirri, Jennifer S., and Barmes, D.E. Epidemiology of oral diseses - differences in national problems. Internat. Dent. J., 29:183-90, Sept. 1979. 